\title{
Serum dietary-related metabolites are predictive of GDM risk in an ethnic specific manner: A multivariate analysis of the Born in Bradford cohort
}

\author{
H. Fuller ${ }^{1}$, J.B. Moore ${ }^{1}$, M.M. Iles ${ }^{2,3}$ and M.A. Zulyniak ${ }^{1}$ \\ ${ }^{1}$ Nutritional Epidemiology Group, School of Food Science and Nutrition, University of Leeds, Leeds, UK, \\ ${ }^{2}$ Leeds Institute of Medical Research, University of Leeds, Leeds, UK and \\ ${ }^{3}$ Leeds Institute for Data Analytics, University of Leeds, Leeds, UK
}

Women of South Asian (SA) descent are at 3-fold greater risk of developing gestational diabetes mellitus (GDM) than those of white European (WE) descent ${ }^{(1)}$, and are more likely to develop type-2 diabetes (T2D) following a GDM diagnosis ${ }^{(2)}$. Despite this, SA-specific determinants of GDM are poorly understood, so require greater attention. This study aims to identify serum metabolites and metabolic patterns in early pregnancy that associate with future GDM within 2688 WE and 2671 SA women in the Born in Bradford $(\mathrm{BiB})$ cohort.

A panel of 146 serum metabolites, comprised of lipoproteins, fatty acids, cholesterol and amino acids, were analysed in blood samples collected prior to maternal GDM assessment. Partial least squares discriminatory analysis (PLSDA) and sparse PLSDA models (accounting for age, BMI, parity, multiple pregnancy and smoking status) were used to identify common and ethnic-specific candidate metabolites and metabolite patterns that are predictive of GDM.

Seven glycolytic, fatty acid, and cholesterol metabolites associated with future GDM diagnosis in both ethnicities (Variable Importance in Projection (VIP) $\geq 1$ ); 6 metabolites (i.e., alanine, glutamine, total serum cholesterol, total PUFAs, omega-6 and citrate) associated with GDM solely in WE women. No SA-specific metabolites were found. Overall, fatty acids were consistently stronger predictors of GDM within WEs compared to SAs. After adjusting for BMI, age, parity, smoking status, and non-singleton pregnancies, $60 \%$ of the variance in GDM case/non-case status was explained in WE women, while only $35 \%$ of the variance was explained in SA women. However, a metabolite profile was uncovered for lean SA women (BMI $\leq 23 \mathrm{~kg} / \mathrm{m} 2)$ that was predictive of subsequent GDM development that was distinct from other SA and WE women metabolic profiles.

Despite a common underlying metabolic profile of GDM risk within our diverse cohort, distinct profiles of metabolites were identified that were predictive of GDM only in WE women or lean SA women. These profiles confirm metabolites previously associated with dysglycemia, insulin sensitivity, inflammation, and modulators of fatty acid metabolism (i.e., alanine, glutamine, PUFAs, and citrate); but go a step further and present ethnic-specific metabolic drivers of GDM. Targeted exploration of these metabolites and their association with GDM offer promising direction for unravelling the aetiology of GDM development and the elevated risk observed in SA women

\section{Acknowledgements}

Born in Bradford is only possible because of the enthusiasm and commitment of the Children and Parents in BiB. We are grateful to all the participants, health professionals and researchers who have made Born in Bradford happen.

\section{References}

1. Mcintyre HD, Catalano P \& Zhang C (2019) Nat Rev Dis Primers 5, 48.

2. Dennison RA, Chen ES, Green ME et al. (2021) Diabetes Res Clin Pract 171, 108625. 